\title{
On non-Newtonian incompressible fluids with phase transitions
}

\author{
Namkwon Kim ${ }^{1}$, Luisa Consiglieri ${ }^{2,3}$ and José Francisco Rodrigues ${ }^{3,4, *, \dagger}$ \\ ${ }^{1}$ School of Mathematical Sciences, Seoul National University, 1649-003 Lisbon, Portugal \\ ${ }^{2}$ CMAF, University of Lisbon, 1649-003 Lisbon, Portugal \\ ${ }^{3}$ Sciences Faculty, University of Lisbon, 1649-003 Lisbon, Portugal \\ ${ }^{4}$ Centro de Matemática da Universidade de Coimbra, Portugal
}

\section{Communicated by B. Straughan}

\begin{abstract}
SUMMARY
A modified model for a binary fluid is analysed mathematically. The governing equations of the motion consists of a Cahn-Hilliard equation coupled with a system describing a class of non-Newtonian incompressible fluid with $p$-structure. The existence of weak solutions for the evolution problems is shown for the space dimension $d=2$ with $p \geqslant 2$ and for $d=3$ with $p \geqslant 11 / 5$. The existence of measure-valued solutions is obtained for $d=3$ in the case $2 \leqslant p<11 / 5$. Similar existence results are obtained for the case of nondifferentiable free energy, corresponding to the density constraint $|\psi| \leqslant 1$. We also give regularity and uniqueness results for the solutions and characterize stable stationary solutions. Copyright (c) 2006 John Wiley \& Sons, Ltd.
\end{abstract}

KEY WORDS: off-normal; simulation; FDTD; metamaterials

\section{INTRODUCTION}

A two-phase flow is fluid motion which has two different phase states. When we consider a two-phase flow between immiscible fluids or a motion of sharp interfaces, it is necessary to take the effect of convection (fluidity) into account together with the free energy of the system. Dynamics of two-phase systems ignoring convection has been studied deeply in the literature and Cahn-Hilliard equation has been playing a central role in this area. NavierStokes equations also have been central in fluid mechanics. Thus, a coupling of Cahn-Hilliard and Navier-Stokes equations can be a first candidate to describe a phase transition phenomena with fluidity when the sharp interface is replaced by a narrow transition layer determined by both diffusion and motion. Indeed, there have been several papers introducing such models to

\footnotetext{
${ }^{*}$ Correspondence to: José Francisco Rodrigues, Universidade de Lisboa/CMAF, Av. Gama Pinto, 2, 1649-003 Lisboa, Portugal.

†E-mail: rodrigue@fc.ul.pt

Contract/grant sponsor: FCT (Fundação para a Ciência e Tecnologia)
}

Copyright @ 2006 John Wiley \& Sons, Ltd.

Received 13 February 2006 
describe fluctuations or hydrodynamic effects in the critical phenomena [1-3]. These models are variations of a model called model $\mathrm{H}$ in Reference [4], which can be systematically derived [5] and generalized [6] or [7] (see also Reference [8]). The model $\mathrm{H}$ is a system of incompressible Navier-Stokes equations coupled with Cahn-Hilliard equation through quadratic coupling terms (reversible modes). The model also encompasses the case of non-constant mobility and surface tension. In particular, for the static case, the model is reduced to one similar in Reference [9]. However, there is a difference between the two models since the model $\mathrm{H}$ admits a variational structure while the model in Reference [9] does not. Phase field models have been also used with success for the numerical computations of interface movement using different methods, as for instance, in Reference [7,10] or [11]. Qualitative studies of the behaviour of Cahn-Hilliard flow model were considered with Navier-Stokes equations $(p=2)$ and constant surface tension coefficient in Reference [12] and also with constant mobility in Reference [13], for slightly nonhomogeneous diphasic incompressible fluids under shear.

In this paper, we consider a convective phase field system for modified model $\mathrm{H}$ on a smooth bounded domain or on a torus for non-Newtonian fluids with $p$-structure. We first introduce the definitions and we prove the existence of weak solutions under relative density (order parameter) dependent viscosity, surface tension coefficient and mobility for $p \geqslant(3 d+2) /(d+2)$ in the space dimensions, $d=2,3$, recovering the Ladyzhenskaya-Lions result [14]. The particular case of Navier-Stokes equations $(p=2)$ is also covered for $d=3$. The Lyapunov functional turns out to fall into the classical case of the Cahn-Hilliard system for the static case. The Lyapunov functional actually guarantees the stability of local minimizers of the classical functional in the absence of external forces.

To fill the gap $2 \leqslant p<11 / 5$ when $d=3$, we prove the existence of measure-valued solutions for $p \geqslant 2(d=2,3)$ in the line of Reference [15]. Then we show the uniqueness of weak solutions for $p \geqslant(d+2) / 2, d=2,3$. Some regularity and existence results are obtained in two-dimensional space $(d=2)$ for a class of non-Newtonian fluids undergoing a well behaved stress tensor with $p$-growth, $p>1$, when the viscosity, surface tension coefficient and mobility are constants. Finally, in the last section, we consider the case of nondifferentiable free energy in order to obtain a solution satisfying the (physical) density constraint $|\psi| \leqslant 1$ as in Reference [16] (see also Reference [17]).

\section{A CONVECTIVE-PHASE FIELD SYSTEM}

The state of the system is described by a pair $(u, \psi)$, where $u=\left(u_{1}(x, t), \ldots, u_{d}(x, t)\right)$ is the velocity field of the fluid and $\psi=\psi(x, t)$ is the order parameter (the relative density). The system of equations for $(u, \psi)$ is

$$
\begin{aligned}
\partial_{t} u+(u \cdot \nabla) u & =-\nabla q+\nabla \cdot(v \tau)-\nabla \cdot(\alpha \nabla \psi \otimes \nabla \psi) \\
\nabla \cdot u & =0 \\
\partial_{t} \psi+u \cdot \nabla \psi & =\nabla \cdot\left(m \nabla\left(f^{\prime}(\psi)-\sqrt{\alpha} \nabla \cdot(\sqrt{\alpha} \nabla \psi)\right)\right)
\end{aligned}
$$

with appropriate initial and boundary conditions. Here, $f \in C^{2}\left(\mathbb{R} \rightarrow \mathbb{R}^{+}\right)$is a volumetric free energy, $v=v(\psi)>0$ the viscosity, $\alpha=\alpha(\psi)>0$ the surface tension coefficient, $m=m(\psi)>0$ the mobility, $q=q(x, t)$ the scalar pressure, and $\tau=\tau(D(u))$ the viscous stress satisfying 
the $(p-1)$-growth and $p$-coercivity conditions, where $D(u)=D_{i j}(u)=\left(\partial_{i} u_{j}+\partial_{j} u_{i}\right) / 2$ is the velocity of strain tensor, $i, j=1, \ldots, d$. System (1)-(3) is derived under the assumption of constant density and incompressibility. We assume that the coefficients $v, \alpha$, and $m$ depend only on $\psi$. We also assume that $v, \alpha$, and $m$ are continuous functions, with $\alpha$ Lipschitz, such that they are bounded from below and above by positive definite constants

$$
0<\gamma_{1} \leqslant v(\psi), \alpha(\psi), m(\psi) \leqslant \gamma_{2} \quad \forall \psi \in \mathbb{R}
$$

These assumptions are reasonable and used in the derivation of (1)-(3) in Reference [5]. Due to physical motivation, we only consider $f$ of double-well type satisfying the following conditions:

$$
\left.\begin{array}{l}
f(y) \geqslant 0, f^{\prime}(y) / f(y)=o(1) \text { as }|y| \rightarrow \infty \\
f(y) \text { has local minima only at } y= \pm 1 \\
f(y) \text { is strictly monotone for }|y|>1
\end{array}\right\}
$$

Denoting by $\mathbb{R}_{\text {sym }}^{d^{2}}$ the set of symmetric $d \times d$ matrices, a non-Newtonian fluid (see Reference [15], for instance) can be described by a monotone $\tau_{i j} \in C\left(\mathbb{R}_{\mathrm{sym}}^{d^{2}}\right)$ such that

$$
\begin{gathered}
\tau_{i j}(0)=0, \quad|\tau(\zeta)| \leqslant \gamma_{3}(1+|\zeta|)^{p-1} \\
\tau(\zeta): \zeta \geqslant \gamma_{4}|\zeta|^{p} \quad \forall \zeta \in \mathbb{R}_{\mathrm{sym}}^{d^{2}}
\end{gathered}
$$

We shall study the initial boundary value problem of the above system on two types of domains, $\Omega_{B}$ and $\Omega_{P} . \Omega_{B} \subset \mathbb{R}^{d}$ is a smooth bounded domain and $\Omega_{P}$ is the usual $d$-torus. For these domains, we work with different boundary conditions

$$
u=\frac{\partial}{\partial n} \psi=\frac{\partial}{\partial n} \mu=0 \quad \text { on } \partial \Omega_{B}
$$

or

$$
u(x)=u(x+\mathbf{e}), \quad \psi(x)=\psi(x+\mathbf{e}), \quad \mu(x)=\mu(x+\mathbf{e}) \quad \text { on } \partial \Omega_{P}
$$

Here, $\mathbf{e}$ is a generic element of a basis of the torus, $n$ is the outward normal vector of $\Omega_{B}$, and

$$
\mu=f^{\prime}(\psi)-\sqrt{\alpha} \nabla \cdot(\sqrt{\alpha} \nabla \psi)
$$

where $\mu$ is called the chemical potential. The boundary condition (8) is physically more meaningful than the Dirichlet-type condition. In fact, we can work with the Dirichlet-type boundary condition, $u=\psi=\Delta \psi=0$ on $\partial \Omega_{B}$ instead of (8) to get similar results. Those boundary conditions can be incorporated in the functional spaces below. First we define the following spaces:

$$
\begin{gathered}
W_{n}^{k, 2}=\left\{\psi \in W^{k, 2} \mid \frac{\partial^{k-1} \psi}{\partial^{k-1} n}=0 \text { on } \partial \Omega_{B}\right\}, \quad k \geqslant 2 \\
\mathscr{V}_{B}\left(\mathscr{V}_{P}\right)=\left\{u \mid u \in\left(C_{0}^{\infty}\right)^{d}\left(\left(C_{\mathrm{per}}^{\infty}\right)^{d}\right), \nabla \cdot u=0\right\} \\
\mathbf{J}_{B}^{k, p}\left(\mathbf{J}_{P}^{k, p}\right)=\left\{u \mid u \in\left(W_{0}^{k, p}\right)^{d}\left(\left(W_{\mathrm{per}}^{k, p}\right)^{d}\right), \nabla \cdot u=0\right\}, \quad k \geqslant 0, \quad p>1
\end{gathered}
$$


Then, interpreting $\mathbf{J}^{0}$ in the generalized sense, the spaces we work with will be

$$
\begin{gathered}
\mathbf{H}_{B}\left(\mathbf{H}_{P}\right)=\mathbf{J}_{B}^{0,2} \times W^{1,2}\left(\mathbf{J}_{P}^{0,2} \times W_{\text {per }}^{1,2}\right) \\
\mathbf{V}_{B}\left(\mathbf{V}_{P}\right)=\mathbf{J}_{B}^{1, p} \times W_{n}^{3,2}\left(\mathbf{J}_{P}^{1, p} \times W_{\text {per }}^{3,2}\right)
\end{gathered}
$$

We denote by $\Omega$ either of the two domains $\Omega_{B}$ and $\Omega_{P}$, and we understand $\mathbf{H}=\mathbf{H}_{B}\left(\mathbf{H}_{P}\right)$ and $\mathbf{V}=\mathbf{V}_{B}\left(\mathbf{V}_{P}\right)$, respectively. Clearly, $\mathbf{H}$ and $\mathbf{V}$ are Banach spaces and $\mathbf{V}$ is compactly embedded in $\mathbf{H}$ for $p>2 d /(d+2)$. We also use the summation convention throughout this paper.

We first define a weak solution of (1)-(3) and afterwards we extend the definition to allow measure-valued solutions.

\section{Definition 1}

We say that $(u, \psi, \mu)$ is a weak solution of (1)-(3) on $\Omega$ for $\left(u_{0}, \psi_{0}\right)$ and $0<t<T$ if $(u, \psi) \in L^{\infty}(0, T ; \mathbf{H}), \nabla u \in L^{p}\left(0, T ; \mathbf{L}^{p}\right), \nabla \mu \in L^{2}\left(0, T ; \mathbf{L}^{2}\right), f(\psi) \in L^{1}\left(0, T ; L^{1}\right)$, and for any test function $(v, \phi) \in \mathbf{V},(u, \psi, \mu)$ satisfies the following formulation:

$$
\begin{aligned}
\int_{\Omega} v \cdot u(t)-\int_{\Omega} v \cdot u_{0} & =\int_{0}^{t} \int_{\Omega}\left(u_{i} u_{j} \partial_{i} v_{j}-v \tau_{i j}(D u) \partial_{i} v_{j}-\psi v \cdot \nabla \mu\right) \\
\int_{\Omega} \phi \psi(t)-\int_{\Omega} \phi \psi_{0} & =\int_{0}^{t} \int_{\Omega}(\psi u \cdot \nabla \phi-m \nabla \phi \cdot \nabla \mu) \\
\int_{\Omega} \mu \phi & =\int_{\Omega}\left(f^{\prime}(\psi) \phi+\sqrt{\alpha} \nabla \psi \cdot \nabla(\sqrt{\alpha} \phi)\right) \quad \text { a.e. } t
\end{aligned}
$$

Note that all terms in (11)-(13) are meaningful. Indeed, $W^{1, p} \hookrightarrow L^{4}$ for $p \geqslant 3 d /(d+2)$ and $d \leqslant 4, \partial_{t} u \in L^{1}\left(0, T ;\left(\mathbf{J}^{1, p}\right)^{\prime}\right)$ for $p \geqslant\left(d+\sqrt{3 d^{2}+4 d}\right) /(d+2)$, and $\partial_{t} u \in L^{p^{\prime}}\left(0, T ;\left(\mathbf{J}^{1, p}\right)^{\prime}\right)$ for $p \geqslant(3 d+2) /(d+2)$. The above equations are formally equivalent to (1)-(3) since

$$
\begin{aligned}
\nabla \cdot(\alpha \nabla \psi \otimes \nabla \psi) & =\sqrt{\alpha} \nabla \cdot(\sqrt{\alpha} \nabla \psi) \nabla \psi+\frac{1}{2} \nabla\left(\alpha|\nabla \psi|^{2}\right) \\
& =-\mu \nabla \psi+\nabla\left(\frac{1}{2} \alpha|\nabla \psi|^{2}+f(\psi)\right)
\end{aligned}
$$

In order to define a measure-valued solution as in Reference [15], we recall the space of probability measures:

$$
\operatorname{Prob}\left(\mathbb{R}^{s}\right) \equiv\left\{\lambda \in \mathscr{M}\left(\mathbb{R}^{s}\right), \lambda \text { non-negative, } \quad \lambda\left(\mathbb{R}^{s}\right)=1\right\}
$$

where $\mathscr{M}\left(\mathbb{R}^{s}\right)$ denotes the space of bounded Radon measures on $\mathbb{R}^{s}$. A mapping $\lambda \in L_{w}^{\infty}\left(\Omega \times(0, T) ; \mathscr{M}\left(\mathbb{R}^{s}\right)\right)$ if and only if

$\lambda: \Omega \times(0, T) \rightarrow \mathscr{M}\left(\mathbb{R}^{s}\right)$ is a weak measurable function, that is, if the function

$$
(x, t) \mapsto\left\langle\lambda_{x, t}, F((x, t), \cdot)\right\rangle=\int_{\mathbb{R}^{s}} F((x, t) ; \eta) \mathrm{d} \lambda_{x, t}(\eta) \quad \forall F \in L^{1}\left(\Omega \times(0, T) ; C_{0}\left(\mathbb{R}^{s}\right)\right)
$$

is measurable. Moreover, the norm

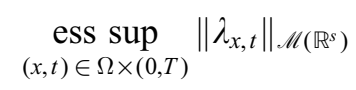

is finite. 


\section{Definition 2}

We say that $(u, \lambda, \psi, \mu)$ is a measure-valued solution of (1)-(3) on $\Omega$ for $\left(u_{0}, \psi_{0}\right)$ and $0<t<T$ if $(u, \psi) \in L^{\infty}(0, T ; \mathbf{H}), \nabla u \in L^{p}\left(0, T ; \mathbf{L}^{p}\right), \lambda \in L_{w}^{\infty}\left(\Omega \times(0, T) ; \operatorname{Prob}\left(\mathbb{R}^{d^{2}}\right)\right), \nabla \mu \in L^{2}\left(0, T ; \mathbf{L}^{2}\right)$, $f(\psi) \in L^{1}\left(0, T ; L^{1}\right)$, and $(u, \lambda, \psi, \mu)$ satisfies the following formulation:

$$
\begin{aligned}
& -\int_{0}^{T} \int_{\Omega} \partial_{t} v \cdot u-\int_{\Omega} v \cdot u_{0} \\
& \quad=\int_{0}^{T} \int_{\Omega}\left(u_{i} u_{j} \partial_{i} v_{j}-v \partial_{i} v_{j} \int_{\mathbb{R}^{d^{2}}} \tau_{i j}\left(\frac{\eta+\eta^{T}}{2}\right) \mathrm{d} \lambda_{x, t}(\eta)-\psi v \cdot \nabla \mu\right)
\end{aligned}
$$

for all $v \in \mathscr{D}((-\infty, T) ; \mathscr{V})$; (12)-(13) for all $\phi \in W_{n}^{3,2}\left(W_{\text {per }}^{3,2}\right)$; and

$$
\partial_{j} u_{i}(x, t)=\int_{\mathbb{R}^{d^{2}}} \eta_{i j} \mathrm{~d} \lambda_{x, t}(\eta) \quad \text { a.e. in } \Omega \times(0, T)
$$

Remark 1

When the measure $\lambda_{x, t}=\left(\delta_{\partial_{j} u_{i}(x, t)}\right)$ is a Dirac measure at almost every point $(x, t) \in \Omega \times(0, T)$ we have

$$
\tau_{i, j}(D(u))=\int_{\mathbb{R}^{d^{2}}} \tau_{i j}\left(\frac{\eta+\eta^{\mathrm{T}}}{2}\right) \mathrm{d} \lambda_{x, t}(\eta)
$$

and a weak solution is also a special measure-valued solution.

\section{EXISTENCE AND LYAPUNOV FUNCTIONAL}

First, we recall a special case of the Gargliardo-Nirenberg inequality [18] which will be used in this paper as

\section{Lemma 1}

Let $i, j$, and $k$ be non-negative integers, $j \leqslant i<k$ and either $v \in H_{0}^{k}\left(\Omega_{B}\right)$ or $v \in H_{\text {per }}^{k}\left(\Omega_{P}\right)$ with $\int_{\Omega_{P}} \nabla^{i} v=0$. Then

$$
\begin{gathered}
\left\|\nabla^{i} v\right\|_{L^{p}} \leqslant C\left\|\nabla^{j} v\right\|_{L^{2}}^{a}\left\|\nabla^{k} v\right\|_{L^{2}}^{1-a}, \quad a=\frac{(2 k-2 i-d) p+2 d}{2(k-j) p} \\
2 \leqslant p \leqslant 2 d /(2 i+d-2 k) \quad \text { if } 2 i+d>2 k \\
2 \leqslant p \leqslant \infty \quad \text { if } 2 i+d<2 k
\end{gathered}
$$

Let us prove some useful a priori estimates.

\section{Lemma 2}

Given $(u, \psi)$, a smooth solution of (1)-(3) with (8) or (9), we have

$$
\begin{gathered}
\int_{\Omega} \psi(t)=\int_{\Omega} \psi(0) \\
Q(t)+2 \int_{0}^{t} \int_{\Omega}\left[v \tau_{i j} \partial_{i} u_{j}+m|\nabla \mu|^{2}\right] \leqslant Q(0)
\end{gathered}
$$


where

$$
Q=\int_{\Omega}\left(u^{2}+2 f(\psi)+\alpha|\nabla \psi|^{2}\right)
$$

and $\mu$ as in (10). As a consequence, for a constant $C>0$

$$
Q(t)+C \int_{0}^{t} \int_{\Omega}\left[|\nabla u|^{p}+|\nabla \mu|^{2}\right] \leqslant Q(0)
$$

Proof

First, (17) can be obtained easily. Indeed, integrating (3) and using the divergence theorem, we recover (17) since the boundary terms vanish due to the boundary conditions. Next, we multiply (1) by $u$ and (3) by $\mu$, then add them after integrating them. Using the divergence theorem, we have

$$
\begin{aligned}
\int_{\Omega} u \cdot \nabla u_{i} u_{i} & =0 \\
\int_{\Omega} \nabla \cdot(v \tau) \cdot u & =-\int_{\Omega} v \tau_{i j} \cdot \partial_{i} u_{j} \\
\int_{\Omega} \partial_{t}\left(2 f(\psi)+|\sqrt{\alpha} \nabla \psi|^{2}\right) & =2 \int_{\Omega} \mu \partial_{t} \psi \\
\int_{\Omega}(u \cdot \nabla \psi) \mu & =\int_{\Omega} \nabla \cdot(f(\psi) u)-\int_{\Omega} u \cdot \sqrt{\alpha} \nabla \psi \nabla \cdot(\sqrt{\alpha} \nabla \psi) \\
& =-\int_{\Omega} u \cdot \nabla \cdot(\alpha \nabla \psi \otimes \nabla \psi)
\end{aligned}
$$

Using the above identities and integrating with respect to the time, we arrive (18). Since $\tau_{i j} \partial_{i} u_{j}=\tau_{i j} D_{i j}(u) \geqslant 0$ then

$$
\int_{\Omega} v \tau_{i j} \partial_{i} u_{j} \geqslant \int_{\Omega} \gamma_{1} \tau_{i j} \partial_{i} u_{j} \geqslant \gamma_{1} \gamma_{4} \int_{\Omega}|\nabla u|^{p}
$$

by assumption (7). Using this fact, we reduce (18) to (19).

In view of Lemma 2, we shall denote from now on

$$
M=\int_{\Omega} \psi_{0}
$$

We shall analyse separately the Navier-Stokes case in three-dimensional space because it is not included in $p \geqslant 11 / 5$ but its linear behaviour in main part still allows the existence of weak solution.

Theorem 1

Given an initial data $\left(u_{0}, \psi_{0}\right) \in \mathbf{H}$ with $f\left(\psi_{0}\right) \in L^{1}$, for $p \geqslant(3 d+2) /(d+2)$ there exists a weak solution $(u, \psi)$ to $(1)-(3)$ for any $T>0$. 


\section{Proof}

We use the Faedo-Galerkin argument. We first show the theorem for $f$ growing at most quadratically near infinity. Let $\left\{\xi_{i}, i \in \mathbb{N}\right\}$ and $\left\{\rho_{i}, i \in \mathbb{N}\right\}$ be an orthogonal basis of $\mathbf{J}^{1, p}$ and $W^{1,2}\left(W_{\text {per }}^{1,2}\right)$, respectively. Clearly, $\rho_{1}=1 /|\Omega|$ and $\left(\xi_{i}, \rho_{j}\right), \quad i, j \in \mathbb{N}$ forms an orthonormal basis for $\mathbf{H}$. And, let $P_{1}^{i}$ and $P_{2}^{i}, i \in \mathbb{N}$ be the projection operators onto span $\left(\xi_{1}, \ldots, \xi_{i}\right)$ and $\operatorname{span}\left(\rho_{1}, \ldots, \rho_{i}\right)$, respectively. We consider the approximate solutions, $\left(u^{i}, \psi^{i}, \mu^{i}\right)$ $\in \operatorname{span}\left(\left(\xi_{1}, \rho_{1}\right), \ldots,\left(\xi_{i}, \rho_{i}\right)\right), i \in \mathbb{N}$ of the following system:

$$
\begin{aligned}
\partial_{t} u^{i}+P_{1}^{i}\left(u^{i} \cdot \nabla u^{i}\right) & =P_{1}^{i}\left(\nabla \cdot\left(v^{i} \tau^{i}\right)\right)+P_{1}^{i}\left(\mu^{i} \nabla \psi^{i}\right) \\
\partial_{t} \psi^{i}+P_{2}^{i}\left(u^{i} \cdot \nabla\right) \psi^{i} & =P_{2}^{i} \nabla \cdot\left(m^{i} \nabla \mu^{i}\right) \\
\mu^{i} & =P_{2}^{i}\left(f^{\prime}\left(\psi^{i}\right)-\sqrt{\alpha^{i}} \nabla \cdot\left(\sqrt{\alpha^{i}} \nabla \psi^{i}\right)\right)
\end{aligned}
$$

where $\alpha^{i}, v^{i}, m^{i}$, and $\tau_{j k}^{i}$ correspond to $\left(u^{i}, \psi^{i}\right)$. We note that $P_{2}^{i}$ in (22) makes the system consistent and is useful to obtain the essential estimates. For any $i \in \mathbb{N}$, the above system is a system of ODEs thus, for the initial data $\left(u_{0}^{i}, \psi_{0}^{i}\right) \equiv\left(P_{1}^{i} u_{0}, P_{2}^{i} \psi_{0}\right)$, the above system has a (local in time) unique solution, $\left(u^{i}, \psi^{i}, \mu^{i}\right)$. Exactly as in Lemma 2 using the idempotency of projection operators, $\left(u^{i}, \psi^{i}, \mu^{i}\right)$ satisfies (18) and $P_{2}^{1} \psi^{i}(t)=P_{2}^{1} \psi_{0}, i \in \mathbb{N}$ like (17). Since $f$ grows at most quadratically in this case,

$$
\int_{\Omega} f\left(\psi_{0}^{i}\right) \leqslant C+C \int_{\Omega}\left|\psi_{0}^{i}\right|^{2} \leqslant C+C \int_{\Omega}\left|\psi_{0}\right|^{2}
$$

Then, $Q\left(u_{0}^{i}, \psi_{0}^{i}\right)(t) \leqslant C Q\left(u_{0}, \psi_{0}\right)$ and thus $Q\left(u^{i}, \psi^{i}\right)(t) \leqslant C Q\left(u_{0}, \psi_{0}\right)$ by (19). By (22),

$$
\begin{aligned}
\left|\int_{\Omega} \mu^{i}\right| & =\left|\int_{\Omega}\left(f^{\prime}\left(\psi^{i}\right)+\sqrt{\alpha^{i}} \nabla \psi^{i} \cdot \nabla \sqrt{\alpha^{i}}\right)\right| \\
& \leqslant C+C \int_{\Omega} f\left(\psi^{i}\right)+C \int_{\Omega}\left|\nabla \psi^{i}\right|^{2} \leqslant C+C Q\left(u_{0}, \psi_{0}\right)
\end{aligned}
$$

Therefore, due to the continuity of the local solution $\left(u^{i}, \psi^{i}, \mu^{i}\right), i \in \mathbb{N}$ and its uniform boundedness in time, we can shift to $T$. Further, for any $T>0,\left(u^{i}, \psi^{i}\right) \in L^{\infty}(0, T ; \mathbf{H}), \nabla u^{i} \in L^{p}$ $\left(0, T ; \mathbf{L}^{p}\right), \nabla \mu^{i} \in L^{2}\left(0, T ; \mathbf{L}^{2}\right)$, and $\mu^{i} \in L^{2}\left(0, T ; W^{1,2}\right)$ uniformly with respect to $i \in \mathbb{N}$.

Next, we multiply (20) and (21) by $v \in \mathbf{J}^{1, p}$ and $\phi \in W^{1,2}\left(W_{\text {per }}^{1,2}\right)$, respectively, to calculate $\left\|\partial_{t} u^{i}\right\|_{\left(\mathbf{J}^{1, p}\right)^{\prime}}$ and $\left\|\partial_{t} \psi^{i}\right\|_{H^{-1}}$. Indeed,

$$
\begin{aligned}
\left|\left\langle\partial_{t} u^{i}, v\right\rangle\right|= & \left|\int_{\Omega} u^{i} \otimes u^{i} \cdot \nabla P_{1}^{i} v-\int_{\Omega} v^{i} \tau^{i} \cdot \nabla P_{1}^{i} v-\int_{\Omega} \psi^{i} P_{1}^{i} v \cdot \nabla \mu^{i}\right| \\
\leqslant & C\left(\left\|u^{i}\right\|_{L^{2 p /(p-1)}}^{2}+\gamma_{2} \gamma_{3}\left(1+\left\|\nabla u^{i}\right\|_{L^{p}}^{p-1}\right)\right)\left\|P_{1}^{i} v\right\|_{W^{1, p}} \\
& +\left\|\psi^{i}\right\|_{L^{4}}\left\|P_{1}^{i} v\right\|_{L^{4}}\left\|\nabla \mu^{i}\right\|_{L^{2}}
\end{aligned}
$$

here, we used the fact, $\nabla \cdot P_{1}^{i} v=0$ and $p \geqslant 3 d /(d+2)$. Subsequently,

$$
\begin{aligned}
\left|\left\langle\partial_{t} u^{i}, v\right\rangle\right| \leqslant & C\left(\left\|u^{i}\right\|_{L^{2}}^{2(1-\beta)}\left\|\nabla u^{i}\right\|_{L^{p}}^{2 \beta}+\gamma_{2} \gamma_{3}\left(1+\left\|\nabla u^{i}\right\|_{L^{p}}^{p-1}\right)\right. \\
& \left.+\left\|\psi^{i}\right\|_{H^{1}}\left\|\nabla \mu^{i}\right\|_{L^{2}}\right)\left\|P_{1}^{i} v\right\|_{W^{1, p}}
\end{aligned}
$$


by the interpolation and Sobolev inequalities for $\beta=d /[(d+2) p-2 d]$. With the fact $\left\|P_{1}^{i} v\right\|_{W^{1, p}}$ $\leqslant\|v\|_{\mathbf{J}^{1, p}}$, (17), the Poincaré inequality, and (19), and chosing $\delta \geqslant 1$ such that $2 \beta \delta \leqslant p$ and $\delta \leqslant p^{\prime}$, we deduce

$$
\int_{0}^{T}\left\|\partial_{t} u^{i}\right\|_{\left(\mathbf{J}^{1, p}\right)^{\prime}}^{\delta} \leqslant C\left(T+M^{4}+Q\left(u_{0}, \psi_{0}\right)^{2 \delta}\right)
$$

for any $T>0$. The limit case $\delta=1$ corresponds to the values $p \geqslant\left(d+\sqrt{3 d^{2}+4 d}\right) /(d+2)$ already found in Reference [15, p. 220].

Considering

$$
\begin{aligned}
\left|\int_{\Omega} \partial_{t} \psi^{i} \phi\right| & =\left|\int_{\Omega} \psi^{i}\left(u^{i} \cdot \nabla\right) P_{2}^{i} \phi-\int_{\Omega} \nabla \cdot\left(P_{2}^{i} \phi\right) m^{i} \nabla \mu^{i}\right| \\
& \leqslant\left(\left\|\psi^{i}\right\|_{L^{4}}\left\|u^{i}\right\|_{L^{4}}+\gamma_{2}\left\|\nabla \mu^{i}\right\|_{L^{2}}\right)\left\|\nabla P_{2}^{i} \phi\right\|_{L^{2}}
\end{aligned}
$$

and applying the Sobolev and Poincare inequalities, (17), and (19), we have

$$
\int_{0}^{T}\left\|\partial_{t} \psi^{i}\right\|_{H^{-1}}^{2} \leqslant C\left(1+M^{4}+Q\left(u_{0}, \psi_{0}\right)^{2}\right)
$$

for any $T>0$. Therefore, for any $T>0$, using a well-known compactness theorem [14], we can find a subsequence of $u^{i}$ converging strongly in $L^{p}\left(0, T ; \mathbf{J}^{0,2}\right)$, since $W^{1, p} \hookrightarrow \hookrightarrow L^{2}$ if $p>2 d /(d+2)$, a subsequence of $\psi^{i}$ converging strongly in $L^{2}\left(0, T ; L^{2}\right)$, and a subsequence of $\mu^{i}$ converging weakly in $L^{2}\left(0, T ; W^{1,2}\right)$. We denote the limits by $u, \psi$, and $\mu$, respectively. Then, for any $T>0, u \in L^{\infty}\left(0, T ; \mathbf{J}^{0,2}\right) \cap L^{p}\left(0, T ; \mathbf{J}^{1, p}\right), \psi \in L^{\infty}\left(0, T ; W^{1,2}\right)$, and Lemma 2 holds for $(u, \psi, \mu)$.

To pass to the limit of the nonlinear term, we refer that the density-dependent coefficient keeps the monotonicity property as

$$
\begin{aligned}
\int_{\Omega}\left(v\left(\psi^{i}\right) \tau^{i}-v\left(\psi^{j}\right) \tau^{j}\right):\left(D^{i}-D^{j}\right)= & \int_{\Omega} v\left(\psi^{i}\right)\left(\tau^{i}-\tau^{j}\right):\left(D^{i}-D^{j}\right) \\
& +\int_{\Omega}\left[v\left(\psi^{i}\right)-v\left(\psi^{j}\right)\right] \tau^{j}:\left(D^{i}-D^{j}\right)
\end{aligned}
$$

for two solutions $\left(u^{i}, \psi^{i}, \mu^{i}\right)$ and $\left(u^{j}, \psi^{j}, \mu^{j}\right)$. Thus applying monotone arguments (see Reference [14]) where the convective term has meaning if and only if $p \geqslant(3 d+2) /(d+2)$ which corresponds to $\delta=p^{\prime}$, the limits satisfy (11) and trivially (12). Since $f^{\prime} / f=o(1)$ and $f(\psi) \in L^{\infty}\left(0, T ; L^{1}\right),(13)$ also holds for $\mu$.

We next consider the case of $f$ growing faster. In this case, we can approximate $f$ by a sequence of $f_{j} \geqslant 0, j \in \mathbb{N}$ growing at most quadratically and satisfying $f_{1} \leqslant f_{2} \leqslant \cdots \leqslant f$. In fact, we can define that $f_{j}(y)=f(y)$ for $|y|<j, f_{j}(y)=1 / 2\left(f_{j}(j)+f_{j}(j+1)\right)$ for $|y|>j+1$, and then make a smooth and monotone interpolation. Then, we have a sequence of solutions $\left(u^{j}, \psi^{j}, \mu^{j}\right)$ for each $f_{j}$ which satisfies all the above results. For each $f_{j}, j \in \mathbb{N}$, $Q\left(u_{0}, \psi_{0}\right)\left(f_{j}\right) \leqslant Q\left(u_{0}, \psi_{0}\right)(f)$ since $f_{j} \leqslant f$. Thus $\left(u^{j}, \psi^{j}, \mu^{j}\right)$ is again a bounded sequence and we can find a limit (up to a subsequence) $(u, \psi, \mu)$ under the same topology as before. By Fatou's lemma, we further deduce $(u, \psi, \mu)$ satisfy (19). The limit is verified to satisfy 
(11) and (12) in a similar fashion as before. Using the fact $f(\psi) \in L^{1}$ and (5), we can also show (13).

The corollary of the above theorem shows that the space of the solution sitting is actually similar to that of the classical Cahn-Hilliard equation.

Corollary 1

Under all the assumptions of the above theorem, suppose further that $\alpha$ is a constant and that

$$
\left.\left|f^{\prime \prime}(y)\right| \leqslant C\left(1+|y|^{r}\right) \quad \begin{array}{ll}
r=3 & \text { if } d=3 \\
\text { for any } r>0 & \text { if } d=2
\end{array}\right\}
$$

Then, the following estimate holds:

$$
\int_{0}^{T}\left\|\nabla^{3} \psi\right\|_{L^{2}}^{2} \leqslant C\left(1+T+M^{8 r /(4-d)}+Q(0)^{4 r /(4-d)}\right) Q(0)
$$

for any $p>1$.

Proof

Considering that all components of the Galerkin system $\rho_{i}, i \in \mathbb{N}$ are eigenvectors of $-\Delta$, we only need to show that $\Delta \nabla \psi \in L^{2}\left(0, T ; \mathbf{L}^{2}\right)$. By (13), $\alpha \Delta \nabla \psi=f^{\prime \prime}(\psi) \nabla \psi-\nabla \mu$. Using (25) and (16), we obtain

$$
\begin{aligned}
\left\|f^{\prime \prime}(\psi) \nabla \psi\right\|_{L^{2}} & \leqslant\left\|f^{\prime \prime}(\psi)\right\|_{L^{2}}\|\nabla \psi\|_{L^{\infty}} \\
& \leqslant C\left(1+\|\psi\|_{L^{2 r}}^{r}\right)\|\nabla \psi\|_{L^{2}}^{1-d / 4}\|\nabla \Delta \psi\|_{L^{2}}^{d / 4}
\end{aligned}
$$

Applying the Poincaré inequality, we have

$$
\|\psi\|_{L^{2 r}} \leqslant C\left(M+\|\nabla \psi\|_{L^{2}}\right)
$$

for both the cases $r=3, d=3$ and any $r>0, d=2$. Then we infer

$$
\|\Delta \nabla \psi\|_{L^{2}} \leqslant C\|\nabla \mu\|_{L^{2}}+C\left(1+M^{4 r /(4-d)}+\|\nabla \psi\|_{L^{2}}^{4 r /(4-d)}\right)\|\nabla \psi\|_{L^{2}}
$$

using the Young inequality. This proves (26) taking into account (18).

Corollary 2

Under all the assumptions of the above corollary, the existence of weak solution remains valid in the case of Newtonian fluids for $\tau=D(u)$ and $d=3$, and we have further $(u, \psi) \in L^{2}(0, T ; \mathbf{V})$ with $p=2$.

Proof

The proof for the case $\tau=D(u)$ and $d=3$ is identical to the proof of Theorem 1 , since we can derive the same estimates for the Galerkin approximations and the weak convergence of $\nabla u^{i}$ to $\nabla u$ in $\mathbf{L}^{2}$ is sufficient to pass to limit $(20)$. Then, we have further $(u, \psi) \in L^{2}(0, T ; \mathbf{V})$ with $p=2$, taking into account the regularity property given at the above corollary. 
Corollary 3

In the absence of the external forces, $(u, \psi) \in \mathbf{H}$ is a stationary stable solution if and only if $u=0$ and $\psi$ is a local minimizer of

$$
Q_{\mathrm{cl}}(\psi)=\int_{\Omega} \alpha|\nabla \psi|^{2}+2 f(\psi)
$$

Proof

As $Q$ is a Lyapunov functional of system (1)-(3), $(0, \psi)$ is a stable stationary solution in $\mathbf{H}$ if $\psi$ is a local minimizer of $Q_{\mathrm{cl}}$. On the contrary, if $\left(u_{1}, \psi_{1}\right)$ is a stable stationary solution in $\mathbf{H}$, we can consider the Cauchy problem with initial data $\left(u_{1}, \psi_{1}\right)$. Then the solution obtained by Theorem 1 must satisfy (18). However, the solution is just $\left(u_{1}, \psi_{1}\right)$, which means $u_{1}=0$ and therefore $Q=Q_{\mathrm{cl}}$. Since $\left(u_{1}, \psi_{1}\right)$ is stable, $\psi_{1}$ is a local minimizer of $Q_{\mathrm{cl}}$.

\section{MEASURE-VALUED SOLUTIONS}

Let us recall first the following consequence of a theorem on Young measures which is the basis to the existence result of measure-valued solutions (cf. [15, Corollary 2.10, p. 172]).

\section{Lemma 3}

Let $Q \subset \mathbb{R}^{d}$ be a bounded open set. Let $z^{i}$ be uniformly bounded in $L^{p}(Q)^{s}$. Then there exists a subsequence still denoted by $z^{i}$ and a measure-valued function $\lambda$, such that, for all $\tau: \mathbb{R}^{s} \rightarrow \mathbb{R}$ satisfying for some $q>0$ the growth condition

$$
|\tau(\eta)| \leqslant C(1+|\eta|)^{q} \quad \forall \eta \in \mathbb{R}^{s}
$$

we have

$$
\tau\left(z^{i}\right) \rightarrow \bar{\tau} \quad \text { in } L^{r}(Q)
$$

where

$$
\bar{\tau}(y)=\left\langle\lambda_{y}, \tau\right\rangle \quad \text { a.e. in } Q
$$

provided that $1<r \leqslant p / q$.

Theorem 2

Given an initial data $\left(u_{0}, \psi_{0}\right) \in \mathbf{H}$ with $f\left(\psi_{0}\right) \in L^{1}$, for $p \geqslant 2,(d=2,3)$, there exists a measurevalued solution $(u, \psi)$ to $(1)-(3)$ for any $T>0$.

\section{Proof}

As in the proof of weak solutions, we first start with the case $f$ growing at most quadratically near infinity. Let $\left\{\xi_{i}, i \in \mathbb{N}\right\}$ and $\left\{\rho_{i}, i \in \mathbb{N}\right\}$ be an orthogonal basis of $\mathbf{J}^{k, 2}, k>1+d / 2$ (cf. Reference $\left[15\right.$, p. 206]), and $W^{1,2}\left(W_{\text {per }}^{1,2}\right)$, respectively. Then there exists an approximate solution $\left(u^{i}, \psi^{i}, \mu^{i}\right), i \in \mathbb{N}$ such that, for any $T>0,\left(u^{i}, \psi^{i}\right) \in L^{\infty}(0, T ; \mathbf{H}), \nabla u^{i} \in L^{p}\left(0, T ; \mathbf{L}^{p}\right)$, $\partial_{t} \psi^{i} \in L^{2}\left(0, T ; H^{-1}\right), \nabla \mu^{i} \in L^{2}\left(0, T ; \mathbf{L}^{2}\right)$, and $\mu^{i} \in L^{2}\left(0, T ; W^{1,2}\right)$ uniformly with respect to $i \in \mathbb{N}$.

However, estimate (23) is not valid for $p<3 d /(d+2)$, that is, $2 \leqslant p<11 / 5$ when $d=3$. In order to prove an estimate for $\partial_{t} u^{i}$ in $L^{p^{\prime}}\left(0, T ;\left(\mathbf{J}^{k, 2}\right)^{\prime}\right)$, we take $v \in L^{p}\left(0, T ; \mathbf{J}^{k, 2}\right)$ such that 
$\|v\|_{L^{p}\left(0, T ; \mathbf{J}^{k, 2}\right)} \leqslant 1$ in (20) it follows

$$
\begin{aligned}
\left|\int_{0}^{T}\left\langle\partial_{t} u^{i}, v\right\rangle \mathrm{d} t\right| \leqslant & C \int_{0}^{T}\left\|u^{i}\right\|_{L^{2}}^{2}\left\|\nabla P_{1}^{i} v\right\|_{L^{\infty}}+\left(1+\left\|\nabla u^{i}\right\|_{L^{p}}^{p-1}\right)\left\|\nabla P_{1}^{i} v\right\|_{L^{p}} \\
& +\left\|\psi^{i}\right\|_{L^{2}}\left\|P_{1}^{i} v\right\|_{L^{\infty}}\left\|\nabla \mu^{i}\right\|_{L^{2}} \mathrm{~d} t \\
\leqslant & C\left(\left\|u^{i}\right\|_{L^{\infty}\left(0, T ; L^{2}\right)}^{2}+1+\left\|\nabla u^{i}\right\|_{L^{p}\left(0, T ; L^{p}\right)}^{p-1}\right. \\
& \left.+\left\|\psi^{i}\right\|_{L^{\infty}\left(0, T ; L^{2}\right)}\left\|\nabla \mu^{i}\right\|_{L^{2}\left(0, T ; L^{2}\right)}\right)\left\|P_{1}^{i} v\right\|_{L^{p}\left(0, T ; W^{k, 2}\right)}
\end{aligned}
$$

remarking that $\left\|P_{1}^{i} v\right\|_{W^{k, 2}} \leqslant\|v\|_{\mathbf{J}^{k, 2}}$ and that $k>1+d / 2$ implies that $\nabla v \in\left(W^{k-1,2}\right)^{d \times d} \hookrightarrow$ $\left(L^{\infty}\right)^{d \times d}$. Then the a priori estimate holds and the limit processes follow as in the proof of Theorem 1, except for the term

$$
\int_{0}^{T} \int_{\Omega} v\left(\psi^{i}\right) \tau^{i}: D(v)
$$

for all $v \in \mathscr{D}(-\infty, T ; \mathscr{V})$. Applying Lemma 3 with $Q=\Omega \times(0, T), z^{i}=D\left(u^{i}\right), q=p-1, r=p^{\prime}$ and $s=d^{2}$, we have

$$
\tau^{i}=\tau\left(D\left(u^{i}\right)\right) \rightarrow \bar{\tau} \quad \text { in } L^{p^{\prime}}(\Omega \times(0, T))^{d^{2}}
$$

where

$$
\bar{\tau}_{i j}(x, t)=\int_{\mathbb{R}^{d^{2}}} \tau_{i j}\left(\frac{\eta+\eta^{\mathrm{T}}}{2}\right) \mathrm{d} \lambda_{x, t}(\eta) \quad \text { a.e. in } \Omega \times(0, T)
$$

Therefore, since $\psi^{i} \rightarrow \psi$ a.e. in $\Omega \times(0, T)$ and $v$ is a continuous function satisfying (4), we conclude

$$
\int_{0}^{T} \int_{\Omega} v\left(\psi^{i}\right) \tau^{i}: D(v) \longrightarrow \int_{0}^{T} \int_{\Omega} v(\psi) \bar{\tau}: D(v)
$$

for all $v \in \mathscr{D}(-\infty, T ; \mathscr{V})$.

Assertion (15) is obtained as in Reference [15, p. 212], that is, applying Lemma 3 with $\tau=\mathrm{id}, q=1, r=p$ and $s=d^{2}$.

\section{UNIQUENESS OF WEAK SOLUTIONS}

In this section, we assume that

$$
\alpha, m, v \text { are positive constants }
$$

and the viscous stress tensor $\tau$ satisfies (6) and, for some constant $\gamma_{5}>0$,

$$
(\tau(\zeta)-\tau(\xi)):(\zeta-\xi) \geqslant \gamma_{5}|\zeta-\xi|^{p} \quad \forall \zeta, \quad \xi \in \mathbb{R}_{\mathrm{sym}}^{d^{2}}
$$

under the restriction $p \geqslant 2$ (cf. Reference [15, p. 198]). Let us prove uniqueness for this case if $d=2$ and for $p \geqslant 5 / 2$ if $d=3$. 
Theorem 3

Assume $p \geqslant(d+2) / 2$ and (25). Then, there exists a unique weak solution for (1)-(3) for a given initial data, $\left(u_{0}, \psi_{0}\right) \in \mathbf{H}$ with $f\left(\psi_{0}\right) \in L^{1}$.

Proof

Let $\left(v_{1}, \psi_{1}\right),\left(v_{2}, \psi_{2}\right)$ be two weak solutions given by Theorem 1 for the same initial data and let $(\bar{v}, \bar{\psi})=\left(v_{1}-v_{2}, \psi_{1}-\psi_{2}\right)$. Since we can take $\phi=1$ in (12), we can assume $\int_{\Omega} \psi_{k}(t)=\int_{\Omega} \psi_{0}$, $k=1$, 2. In particular, $\int_{\Omega} \bar{\psi}=0$. Similarly, $\int_{\Omega} \bar{v}=0$ when $\Omega=\Omega_{P}$. This fact allows the application of (16) for $i=j=0$ in several occasions. We subtract the equations for $\left(v_{2}, \psi_{2}\right)$ from $\left(v_{1}, \psi_{1}\right)$ and integrate them after multiplying by $(\bar{v}, \bar{\psi})$ to obtain

$$
\begin{aligned}
& \partial_{t} \int_{\Omega}|\bar{\psi}|^{2}+2 m \alpha \int_{\Omega}|\Delta \bar{\psi}|^{2} \leqslant 2 m \int_{\Omega}|\Delta \bar{\psi}|\left|f^{\prime}\left(\psi_{1}\right)-f^{\prime}\left(\psi_{2}\right)\right|+2 \int_{\Omega}\left|\bar{v} \cdot \nabla \psi_{2} \bar{\psi}\right| \\
& \leqslant C\|\Delta \bar{\psi}\|_{L^{2}}\left\|f^{\prime}\left(\psi_{1}\right)-f^{\prime}\left(\psi_{2}\right)\right\|_{L^{2}}+C\|\bar{v}\|_{L^{2}}\left\|\nabla \psi_{2}\right\|_{L^{\infty}}\|\bar{\psi}\|_{L^{2}} \\
& \partial_{t} \int_{\Omega}|\bar{v}|^{2}+2 v \gamma_{5} \int_{\Omega}|\nabla \bar{v}|^{2} \leqslant 2 \alpha \int_{\Omega}\left(\left|\nabla \Delta \psi_{1}\right||\bar{v} \bar{\psi}|+|\Delta \bar{\psi}|\left|\nabla \psi_{2}\right||\bar{v}|\right)+2 \int_{\Omega}\left|\bar{v} \otimes \bar{v}: \nabla v_{2}\right| \\
& \leqslant C\left\|\nabla \Delta \psi_{1}\right\|_{L^{2}}\|\bar{v} \bar{\psi}\|_{L^{2}}+\epsilon \int_{\Omega}|\Delta \bar{\psi}|^{2} \\
& +C_{\epsilon}\|\bar{v}\|_{L^{2}}^{2}\left\|\nabla \psi_{2}\right\|_{L^{\infty}}^{2}+C\left\|\nabla v_{2}\right\|_{L^{p}}\|\bar{v}\|_{L^{2 p /(p-1)}}^{2}
\end{aligned}
$$

here, $\epsilon>0$ is arbitrary. Using the mean value theorem and (16), we have

$$
\left\|f^{\prime}\left(\psi_{1}\right)-f^{\prime}\left(\psi_{2}\right)\right\|_{L^{2}} \leqslant\|\bar{\psi}\|_{L^{4}}\left\|f^{\prime \prime}(\xi)\right\|_{L^{4}} \leqslant C\|\bar{\psi}\|_{L^{2}}^{3 / 4}\|\Delta \bar{\psi}\|_{L^{2}}^{1 / 4}\left\|f^{\prime \prime}(\xi)\right\|_{L^{4}}
$$

for some measurable $\xi(x) \in\left[\psi_{1}(x), \psi_{2}(x)\right]$ a.e. $x \in \Omega$. While,

$$
\begin{aligned}
\left\|\nabla \Delta \psi_{1}\right\|_{L^{2}}\|\bar{v} \bar{\psi}\|_{L^{2}} \leqslant & \left\|\nabla \Delta \psi_{1}\right\|_{L^{2}}\|\bar{v}\|_{L^{4}}\|\bar{\psi}\|_{L^{4}} \\
\leqslant & \left\|\nabla \Delta \psi_{1}\right\|_{L^{2}}\|\bar{v}\|_{L^{2}}^{1 / 2}\|\nabla \bar{v}\|_{L^{2}}^{1 / 2}\|\bar{\psi}\|_{L^{2}}^{3 / 4}\|\Delta \bar{\psi}\|_{L^{2}}^{1 / 4} \\
\leqslant & C_{\epsilon}\left\|\nabla \Delta \psi_{1}\right\|_{L^{2}}\|\bar{v}\|_{L^{2}}^{2}+C_{\epsilon}\left\|\nabla \Delta \psi_{1}\right\|_{L^{2}}^{2}\|\bar{\psi}\|_{L^{2}}^{2} \\
& +\epsilon\|\Delta \bar{\psi}\|_{L^{2}}^{2}+\epsilon\|\nabla \bar{v}\|_{L^{2}}^{2}
\end{aligned}
$$

and

$$
\begin{aligned}
\left\|\nabla v_{2}\right\|_{L^{p}}\|\bar{v}\|_{L^{2 p /(p-1)}}^{2} & \leqslant\left\|\nabla v_{2}\right\|_{L^{p}}\|\bar{v}\|_{L^{2}}^{(2 p-d) / p}\|\nabla \bar{v}\|_{L^{2}}^{d / p} \\
& \leqslant C_{\epsilon}\left\|\nabla v_{2}\right\|_{L^{2}}^{2 p /(2 p-d)}\|\bar{v}\|_{L^{2}}^{2}+\epsilon\|\nabla \bar{v}\|_{L^{2}}^{2}
\end{aligned}
$$

Then, taking $\epsilon$ small enough and denoting $A=\|\bar{v}\|_{L^{2}}^{2}+\|\bar{\psi}\|_{L^{2}}^{2}$, we obtain

$$
\partial_{t} A \leqslant C A\left(1+\left\|\nabla \psi_{2}\right\|_{L^{\infty}}^{2}+\left\|f^{\prime \prime}(\xi)\right\|_{L^{4}}^{8 / 3}+\left\|\nabla \Delta \psi_{1}\right\|_{L^{2}}^{2}+\left\|\nabla v_{2}\right\|_{L^{2}}^{2 p /(2 p-d)}\right)
$$


Due to the assumption on $f$, we have

$$
\begin{aligned}
\left\|f^{\prime \prime}(\xi)\right\|_{L^{4}} & \leqslant C\left(1+\left\|\left.\xi\right|^{r}\right\|_{L^{4}}\right) \leqslant C\left(1+\left\|\psi_{1}\right\|_{L^{4 r}}^{r}+\left\|\psi_{2}\right\|_{L^{4 r}}^{r}\right) \\
& \leqslant C\left(1+\left\|\nabla \psi_{1}\right\|_{L^{2}}^{r}+\left\|\nabla \psi_{2}\right\|_{L^{2}}^{r}+M^{r}\right)
\end{aligned}
$$

and due to (26) and (18), we obtain

$$
1+\left\|\nabla \psi_{2}\right\|_{L^{\infty}}^{2}+\left\|f^{\prime \prime}(\xi)\right\|_{L^{4}}^{8 / 3}+\left\|\nabla \Delta \psi_{1}\right\|_{L^{2}}^{2}+\left\|\nabla v_{2}\right\|_{L^{2}}^{2 p /(2 p-d)} \in L^{1}(0, T)
$$

where we take into account that $2 p /(2 p-d) \leqslant p$ is equivalent to the assumption $p \geqslant(d+2) / 2$. Therefore, we can apply the Grönwall lemma and conclude $A(t)=0$ for all $t>0$.

\section{REGULARITY IN TWO DIMENSIONS}

In this section, we assume that

$$
\alpha, m, v \text { are positive constants, and } d=2
$$

Now the viscous stress tensor is described by a differentiable functional, that is, there exists a strictly convex potential $U \in C^{2}\left(\mathbb{R}_{\mathrm{sym}}^{4}\right)$ of $\tau$ such that, for some $p \in(1, \infty)$ and positive constants $\gamma_{6}$ and $\gamma_{7}$,

$$
\begin{gathered}
\tau_{i j}(\zeta)=\frac{\partial U}{\partial \zeta_{i j}}(\zeta): \quad U(0)=\frac{\partial U}{\partial \zeta_{i j}}(0)=0 \\
\frac{\partial^{2} U}{\partial \zeta_{i j} \partial \zeta_{k l}}(\zeta) \xi_{i j} \xi_{k l} \geqslant \gamma_{6}(1+|\zeta|)^{p-2}|\xi|^{2} \\
\left|\frac{\partial^{2} U}{\partial \zeta_{i j} \partial \zeta_{k l}}(\zeta)\right| \leqslant \gamma_{7}(1+|\zeta|)^{p-2} \quad \forall \zeta, \xi \in \mathbb{R}_{\mathrm{sym}}^{4}
\end{gathered}
$$

For $U(D(u))=|D(u)|^{2}$, system (1) reduces to the Navier-Stokes equations. When $f^{\prime \prime}(y)=O\left(|y|^{r}\right), r<\infty$ as in Corollary 1 , then $(u, \psi)(t) \in \mathbf{V}$ a.e. in time. We shall show that in fact, $(u, \psi)(t) \in \mathbf{V}$ for all $t>0$ and the solution is then the unique strong solution.

\section{Lemma 4}

Let $(u, \psi)$ be the weak solution we have found for $p \geqslant 2$. If further $u_{0} \in \mathbf{J}^{1,2}$ and (25) holds in the case $d=2$, then $u \in L^{\infty}\left(0, T ; \mathbf{J}^{1,2}\right)$ and for any $0<t<T$

$$
\|\nabla u\|_{L^{2}}^{2}(t)+v \gamma_{6} \int_{0}^{t}\left\|\nabla^{2} u\right\|_{L^{2}}^{2} \leqslant\left\|\nabla u_{0}\right\|_{L^{2}}^{2}+C\left(1+T+M^{4 r}+Q(0)^{2 r}\right) Q(0)^{2}
$$

Proof

Considering the Galerkin system (20)-(22) for the eigenvectors of the Stokes operator in $W^{2,2}(\Omega)^{2}$, we can multiply the $i$ th equation by $\lambda_{i} \xi_{i}(t)$, where $\lambda_{i}$ are the corresponding 
eigenvalues, and sum over $i \in \mathbb{N}$. Thus, we can suppose $u$ is smooth enough and do a priori estimate. Multiplying Equation (1) by $\Delta u$ and integrating it,

$$
\frac{1}{2} \partial_{t} \int_{\Omega}|\nabla u|^{2}+v \gamma_{6} \int_{\Omega}(1+|\nabla u|)^{p-2}\left|\nabla^{2} u\right|^{2} \leqslant \alpha \int_{\Omega}|\Delta \psi||\nabla \psi||\Delta u|
$$

taking into account

$$
-\int_{\Omega}(u \cdot \nabla) u \Delta u=\int_{\Omega} \partial_{k} u_{j} \partial_{j} u_{i} \partial_{k} u_{i}+\int_{\Omega} u_{j} \partial_{j k} u_{i} \partial_{k} u_{i}=0
$$

Indeed, each term vanishes for $d=2$ using (2). For $p \geqslant 2$

$$
\frac{1}{2} \partial_{t} \int_{\Omega}|\nabla u|^{2}+v \gamma_{6} \int_{\Omega}\left|\nabla^{2} u\right|^{2} \leqslant C\|\Delta \psi\|_{L^{2}}^{2}\|\nabla \psi\|_{L^{\infty}}^{2}+\frac{v \gamma_{6}}{2}\left\|\nabla^{2} u\right\|_{L^{2}}^{2}
$$

By (16), we have

$$
\partial_{t} \int_{\Omega}|\nabla u|^{2}+v \gamma_{6} \int_{\Omega}\left|\nabla^{2} u\right|^{2} \leqslant C\|\nabla \psi\|_{L^{2}}^{2}\left\|\nabla^{3} \psi\right\|_{L^{2}}^{2}
$$

Then (18), (26), and the above inequality imply (31), completing the proof.

\section{Lemma 5}

If $p>1, u_{0} \in \mathbf{J}^{1,2}, \psi_{0} \in W^{2,2}, f \in C^{3}$, and $f^{\prime \prime}, f^{\prime \prime \prime}$ both satisfy the growth condition (25) in case $d=2$, then a solution for (1)-(3) satisfies

$$
\begin{aligned}
\|\Delta \psi\|_{L^{2}}^{2}(t)+m \alpha \int_{0}^{t}\left\|\Delta^{2} \psi\right\|_{L^{2}}^{2} \leqslant & \left\|\Delta \psi_{0}\right\|_{L^{2}}^{2}+C\left[Q(0)^{2}+\right. \\
& \left.\left(1+T+M^{6 r}+Q(0)^{3 r}\right)\left(Q(0)+Q(0)^{2}+Q(0)^{4}\right)\right]
\end{aligned}
$$

Moreover, $u \in L^{\infty}\left(0, T ; \mathbf{J}^{1,2}\right) \cap L^{2}\left(0, T ; \mathbf{J}^{2, p}\right)$ for $p<2$.

Proof

We apply $\Delta$ to (3) and multiply it by $\Delta \psi$. Then integrating it using divergence theorem, we have

$$
\begin{aligned}
\partial_{t} \int_{\Omega}|\Delta \psi|^{2} & \leqslant-2 m \alpha \int_{\Omega}\left|\Delta^{2} \psi\right|^{2}+\int_{\Omega}\left|u-M_{u}\right||\nabla \psi|\left|\Delta^{2} \psi\right|+2 \int_{\Omega}\left|\Delta f^{\prime}(\psi)\right|\left|\Delta^{2} \psi\right| \\
& \leqslant-m \alpha \int_{\Omega}\left|\Delta^{2} \psi\right|^{2}+C \int_{\Omega}\left(\left|u-M_{u}\right|^{2}|\nabla \psi|^{2}+\left|\Delta f^{\prime}(\psi)\right|^{2}\right)
\end{aligned}
$$

Here, $M_{u}=1 /|\Omega| \int_{\Omega} u$ is added since

$$
\int_{\Omega} \Delta\left(M_{u} \cdot \nabla \psi\right) \Delta \psi=\frac{1}{2} \int_{\Omega} M_{u} \cdot \nabla|\Delta \psi|^{2}=0
$$


By (16) and the Poincaré inequality,

$$
\begin{aligned}
\int_{\Omega}\left|u-M_{u}\right|^{2}|\nabla \psi|^{2} \leqslant & \left\|u-M_{u}\right\|_{L^{2}}^{2}\|\nabla \psi\|_{L^{\infty}}^{2} \leqslant C\|u\|_{L^{2}}^{2}\|\nabla \psi\|_{L^{2}}\left\|\nabla^{3} \psi\right\|_{L^{2}} \\
\int_{\Omega}\left|\Delta f^{\prime}(\psi)\right|^{2} \leqslant & \int_{\Omega}\left(\left|f^{\prime \prime}(\psi)\right|^{2}|\Delta \psi|^{2}+\left|f^{\prime \prime \prime}(\psi)\right|^{2}|\nabla \psi|^{4}\right) \\
\leqslant & C\left(1+\|\psi\|_{L^{2 r}}^{2 r}\right)\left(\|\Delta \psi\|_{L^{\infty}}^{2}+\|\nabla \psi\|_{L^{\infty}}^{4}\right) \\
\leqslant & C\left(1+M^{2 r}+\|\nabla \psi\|_{L^{2}}^{2 r}\right)\left(\|\nabla \psi\|_{L^{2}}^{2 / 3}\left\|\nabla^{4} \psi\right\|_{L^{2}}^{4 / 3}\right. \\
& \left.+\|\nabla \psi\|_{L^{2}}^{8 / 3}\left\|\nabla^{4} \psi\right\|_{L^{2}}^{4 / 3}\right)
\end{aligned}
$$

Rearranging the terms and using (18), applying Young inequality and (26), we obtain

$$
\begin{aligned}
\partial_{t} \int_{\Omega}|\Delta \psi|^{2}+m \alpha \int_{\Omega}\left|\Delta^{2} \psi\right|^{2} \leqslant & C\left(\|u\|_{L^{2}}^{4}+\|\nabla \psi\|_{L^{2}}^{2}\left\|\nabla^{3} \psi\right\|_{L^{2}}^{2}\right) \\
& +C\left(1+M^{2 r}+\|\nabla \psi\|_{L^{2}}^{2 r}\right)^{3}\left(\|\nabla \psi\|_{L^{2}}^{2}+\|\nabla \psi\|_{L^{2}}^{8}\right)
\end{aligned}
$$

Integrating with respect to time, we recover the inequality.

To prove that $u \in L^{\infty}\left(0, T ; \mathbf{J}^{1,2}\right) \cap L^{2}\left(0, T ; \mathbf{J}^{2, p}\right)$, we argue as in Lemma 4 to obtain (32). Applying the result given in $[15$, p. 227] for $1<p<2$

$$
\left\|\nabla^{2} u\right\|_{L^{p}}^{2} \leqslant C \mathscr{I}_{p}(u)\left(1+\|\nabla u\|_{p}\right)^{2-p}
$$

for some constant $C>0$ and $\mathscr{I}_{p}(u)=\int_{\Omega}(1+|\nabla u|)^{p-2}\left|\nabla^{2} u\right|^{2}$. Hence, we obtain

$$
\begin{aligned}
\frac{1}{2} \partial_{t} \int_{\Omega}|\nabla u|^{2}+v \gamma_{6} \mathscr{I}_{p}(u) & \leqslant \alpha\|\Delta \psi\|_{L^{p^{\prime}}}\|\nabla \psi\|_{L^{\infty}}\left\|\nabla^{2} u\right\|_{L^{p}} \\
& \leqslant C\|\Delta \psi\|_{L^{p^{p}}}\|\nabla \psi\|_{L^{\infty}} \mathscr{I}_{p}^{1 / 2}(u)\left(1+\|\nabla u\|_{p}\right)^{(2-p) / 2}
\end{aligned}
$$

By Hölder inequality, we have

$$
\partial_{t} \int_{\Omega}|\nabla u|^{2}+v \gamma_{6} \mathscr{I}_{p}(u) \leqslant C\|\Delta \psi\|_{L^{p^{\prime}}}^{2}\|\nabla \psi\|_{L^{\infty}}^{2}\left(1+\|\nabla u\|_{p}\right)^{2-p}
$$

Integrating in time this yields

$$
\|\nabla u\|_{L^{2}}^{2}(t)+v \gamma_{6} \int_{0}^{t} \mathscr{I}_{p}(u) \leqslant\left\|\nabla u_{0}\right\|_{L^{2}}^{2}+C \int_{0}^{t}\|\Delta \psi\|_{L^{p^{\prime}}}^{p^{\prime}}\|\nabla \psi\|_{L^{\infty}}^{p^{\prime}}+\int_{0}^{t}\left(1+\|\nabla u\|_{p}\right)^{p}
$$

Then using the regularity for $\psi,(19)$ and rewriting (33) as

$$
\int_{0}^{T}\left\|\nabla^{2} u\right\|_{L^{p}}^{2} \leqslant\left(T+\sup _{t \in[0, T]}\|\nabla u\|_{L^{2}}^{2}(t)\right) \int_{0}^{T} \mathscr{I}_{p}(u)
$$

the required result follows. 
As a direct consequence of the above two lemmas, the weak solution we have obtained satisfy almost everywhere the equations. From Lemmas 4 and 5 and Theorem 3, we can state the following regularity theorem.

Theorem 4

For $p>1, u_{0} \in \mathbf{J}^{1,2}, \psi_{0} \in W^{2,2}$ with $f\left(\psi_{0}\right) \in L^{1}$, there exists a solution in

$$
\begin{array}{ll}
L^{\infty}\left(0, T ; \mathbf{J}^{1,2} \times W^{2,2}\right) \cap L^{2}\left(0, T ; \mathbf{J}^{2,2} \times W^{4,2}\right) & \text { if } p \geqslant 2 \\
L^{\infty}\left(0, T ; \mathbf{J}^{1,2} \times W^{2,2}\right) \cap L^{2}\left(0, T ; \mathbf{J}^{2, p} \times W^{4,2}\right) & \text { if } p<2
\end{array}
$$

and the solution belongs to $\mathbf{V}(p=2)$ as soon as $t>0$ if $f \in C^{3}$ and $f^{\prime \prime}, f^{\prime \prime \prime}$ both satisfy (25) in case $d=2$. Moreover, this solution is unique if $p \geqslant 2$.

Proof

For $p \geqslant 2$, denoting by $(u, \psi)$ the unique solution given by Theorem 3 , Lemmas 4 and 5 guarantee that this solution belongs to $\mathbf{J}^{1,2} \times W^{2,2}$ for any $t>0$. For any $0<t_{1}<t_{2}, u(t)$, $\nabla \psi(t) \in\left(L^{q}\right)^{2}, \forall q>1$ and $\psi(t) \in L^{\infty}$ uniformly with respect to $t \in\left[t_{1}, t_{2}\right]$. Thus

$$
\partial_{t} \psi+\Delta^{2} \psi=u \cdot \nabla \psi+\Delta f^{\prime}(\psi) \equiv h \in L^{2}\left(t_{1}, t_{2} ; L^{2}\right)
$$

Therefore, $\psi \in W^{3,2}$ for $t_{1}<t<t_{2}$, which finishes the proof for $p \geqslant 2$.

For $1<p<2$, arguing as in the proof of Theorem 2 with $k=2$, we obtain an approximate solution $\left(u^{i}, \psi^{i}, \mu^{i}\right) i \in \mathbb{N}$ such that, for any $T>0,\left(u^{i}, \psi^{i}\right) \in L^{\infty}(0, T ; \mathbf{H}), \nabla u^{i} \in L^{p}\left(0, T ;\left(L^{p}\right)^{4}\right)$, $\partial_{t} \psi^{i} \in L^{2}\left(0, T ; H^{-1}\right), \nabla \mu^{i} \in L^{2}\left(0, T ;\left(L^{2}\right)^{2}\right)$, and $\mu^{i} \in L^{2}\left(0, T ; W^{1,2}\right)$ uniformly with respect to $i \in \mathbb{N}$; and $\partial_{t} u^{i}$ belongs to a bounded set of

$$
L^{\gamma}\left(0, T ;\left(\left(W^{2,2}\right)^{2} \cap \mathbf{J}^{1, p}\right)^{\prime}\right)
$$

with $\gamma=\min (p, 2(p-1))$. Indeed,

$$
\begin{aligned}
\left|\left\langle\partial_{t} u^{i}, v\right\rangle\right| \leqslant & \left\|u^{i}\right\|_{L^{p^{p}}}\left\|\nabla u^{i}\right\|_{L^{p}}\left\|P_{1}^{i} v\right\|_{L^{\infty}}+\gamma_{2} \gamma_{7}\left(1+\left\|\nabla u^{i}\right\|_{L^{p}}\right)^{p-1}\left\|\nabla P_{1}^{i} v\right\|_{L^{p}} \\
& +\alpha\|\Delta \psi\|_{L^{2}}\|\nabla \psi\|_{L^{2}}\left\|P_{1}^{i} v\right\|_{L^{\infty}} \\
= & I_{1}+I_{2}+I_{3}
\end{aligned}
$$

Let us estimate $I_{1}, I_{2}$ and $I_{3}$ separately. Due to the interpolation inequality (see Reference $[15$, p. 232], for instance)

$$
\|u\|_{L^{p^{\prime}}} \leqslant\|u\|_{L^{2}}^{(3 p-4) / 2(p-1)}\|u\|_{L^{2 p /(2-p)}}^{(2-p) / 2(p-1)}
$$

we have

$$
\begin{aligned}
\int_{0}^{T} I_{1} \mathrm{~d} t & \leqslant\left\|u^{i}\right\|_{L^{\infty}\left(0, T ; L^{2}\right)} \int_{0}^{T}\left\|\nabla u^{i}\right\|_{L^{p}}^{1+((2-p) / 2(p-1))}\left\|P_{1}^{i} v\right\|_{W^{2,2}} \mathrm{~d} t \\
& \leqslant C\left\|u^{i}\right\|_{L^{p}\left(0, T ; \mathbf{J}^{1, p}\right)}^{1 / 2(p-1)}\|v\|_{L^{2(p-1) /(2 p-3)\left(0, T ; W^{2,2}\right)}}
\end{aligned}
$$


The second one yields

$$
\begin{aligned}
\int_{0}^{T} I_{2} \mathrm{~d} t & \leqslant C \int_{0}^{T}\left(1+\left\|\nabla u^{i}\right\|_{L^{p}}\right)^{p-1}\|v\|_{W^{2,2}} \mathrm{~d} t \\
& \leqslant C\left(1+\left\|u^{i}\right\|_{L^{p}\left(0, T ; \mathbf{J}^{1, p}\right)}^{p-1}\right)\|v\|_{L^{p}\left(0, T ; W^{2,2}\right)}
\end{aligned}
$$

taking into account that $\left(W^{2,2}\right)^{2} \hookrightarrow\left(W^{1, p}\right)^{2}$ if $p>1$.

From Lemma 5, we obtain

$$
\int_{0}^{T} I_{3} \mathrm{~d} t \leqslant C\|\Delta \psi\|_{L^{\infty}\left(0, T ; L^{2}\right)}\|\nabla \psi\|_{L^{\infty}\left(0, T, L^{2}\right)} \int_{0}^{T}\|v\|_{W^{2,2}} \mathrm{~d} t
$$

Hence, $\partial_{t} u^{i} \in L^{\gamma}\left(0, T ;\left(\left(W^{2,2}\right)^{2} \cap \mathbf{J}^{1, p}\right)^{\prime}\right), \quad u^{i} \in L^{2}\left(0, T ; \mathbf{J}^{2, p}\right) \quad p<2$ and $\mathbf{J}^{2, p} \hookrightarrow \hookrightarrow\left(W^{1, p}\right)^{2}$, $p \geqslant 1$, then

$$
\nabla u^{i} \rightarrow \nabla u \quad \text { a.e. in } \Omega \times(0, T)
$$

and also (since $\tau_{i j} \in C^{1}\left(\mathbb{R}_{\text {sym }}^{4}\right)$ )

$$
\tau\left(D\left(u^{i}\right)\right) \rightarrow \tau(D(u)) \quad \text { a.e. in } \Omega \times(0, T)
$$

By standard arguments [15, p. 224], this implies

$$
\int_{0}^{T} \int_{\Omega} v^{i} \tau^{i}: D(v) \rightarrow \int_{0}^{T} \int_{\Omega} v \tau: D(v) \mathrm{d} x \mathrm{~d} t
$$

\section{NON-DIFFERENTIABLE CASE}

In order $((1)-(3))$ to model a phase transition phenomena, since $\psi(x, t)$ indicates the phase of the system at $(x, t)$, it is required that $|\psi| \leqslant 1$. In this section, we show that $|\psi| \leqslant 1$ may be obtained by using a standard penalization scheme for a non-differentiable free energy $[16,17]$. Let $f=f_{1}+f_{2} ; f_{1}$ satisfies (5) and

$$
f_{2}(y)= \begin{cases}0 & \text { for }|y| \leqslant 1 \\ +\infty & \text { for }|y|>1\end{cases}
$$

The subdifferential of $f_{2}$ is denoted by $\partial f_{2}$, and we set

$$
f^{\prime}(y)=\left\{f_{1}^{\prime}(y)+\chi \mid \chi \in \partial f_{2}(y)\right\}
$$

\section{Definition 3}

We say that $(u, \psi, \mu)$ is a generalized solution for (1)-(3) if for any $(v, \phi) \in \mathbf{V}$ with $f_{2}(\phi) \in L^{1}$, $(u, \psi, \mu)$ satisfy the definition of a weak solution with (13) replaced by

$$
\begin{gathered}
\int_{\Omega}\left(\left(\mu-f_{1}^{\prime}(\psi)\right)(\psi-\phi)-\sqrt{\alpha} \nabla \psi \cdot \nabla(\sqrt{\alpha}(\psi-\phi))\right) \\
\geqslant \int_{\Omega}\left(f_{2}(\psi)-f_{2}(\phi)\right) \text { a.e. } t
\end{gathered}
$$




\section{Theorem 5}

Given $\left(u_{0}, \psi_{0}\right) \in \mathbf{H}$ with $\left|\psi_{0}\right| \leqslant 1$ and $f$ as above, for $p \geqslant(3 d+2) /(d+2)$ if $d=2,3$ or in the Newtonian case $\tau=D(u)$ with $p=2$ and $d=3$, there exists a generalized solution of (1)-(3) provided

$$
(1+|y|)\left|\alpha^{\prime}(y)\right| \leqslant 2 \alpha(y)
$$

Proof

We first introduce the following approximating sequence of $f_{2}$ :

$$
\begin{aligned}
& f_{2}^{j}(y)=0 \text { for }|y|<1 \\
& f_{2}^{j}(y)=j\left(|y|^{2}-1\right)^{2} \text { for }|y| \geqslant 1
\end{aligned}
$$

Now, we consider the initial boundary problem (1)-(3) with $f^{j} \equiv f_{1}+f_{2}^{j}$. There exists a weak solution corresponding to $f^{j}$ by Theorem 1 . We denote by $\left(u^{j}, \psi^{j}, \mu^{j}\right)$ the corresponding solutions. Since $Q\left(u_{0}, \psi_{0}\right)\left(f^{j}\right) \leqslant Q\left(u_{0}, \psi_{0}\right)(f)<\infty$, repeating the argument of Theorem 1, we deduce that $\left(u^{j}, \psi^{j}\right), j \in \mathbb{N}$ is a bounded sequence in $L^{\infty}(0, T ; \mathbf{H}), u^{j}$ in $L^{p}\left(0, T ; \mathbf{J}^{1, p}\right)$, and $\mu^{j}$ in $L^{2}\left(0, T ; W^{1,2}\right)$ for any $T>0$. Also, $\partial_{t} u^{j} \in L^{1}\left(0, T ;\left(\mathbf{J}^{1, p}\right)^{\prime}\right)$ and $\partial_{t} \psi^{j} \in L^{2}\left(0, T ; H^{-1}\right)$ uniformly with respect to $j$ by (23) and (24). Thus, as $j \rightarrow \infty$ a subsequence of $\left(u^{j}, \psi^{j}, \mu^{j}\right)$ converges to $(u, \psi, \mu)$ strongly in $L^{2}\left(0, T ; \mathbf{J}^{0,2} \times L^{2} \times L^{2}\right)$ and weakly in $L^{2}\left(0, T ; \mathbf{H} \times W^{1,2}\right)$. It is easy to show that $(u, \psi, \mu)$ satisfies $(11)$ and (12) as in Theorem 1. Due to the lower semicontinuity of norms and the Fatou's lemma, $(u, \psi, \mu)$ satisfies (18) with $f$. As a consequence, $|\psi| \leqslant 1$ for all $t \in[0, T]$. Finally, we show (34). Without loss of generality, we can assume $f_{1}^{\prime}(y)=O\left(|y|^{2}\right)$ as in the proof of Theorem 1. Then, for $\phi \in W^{3,2}$ with $f(\phi) \in L^{1}$,

$$
\begin{gathered}
\int_{\Omega}\left(\mu-f_{1}^{\prime}(\psi)\right)(\psi-\phi)=\lim _{j} \int_{\Omega}\left(\mu^{j}-f_{1}^{\prime}\left(\psi^{j}\right)\right)\left(\psi^{j}-\phi\right) \quad \text { a.e. } t \\
\int_{\Omega}\left(f_{2}(\psi)-f_{2}(\phi)\right) \leqslant \liminf _{j} \int_{\Omega}\left(f_{2}^{j}\left(\psi^{j}\right)-f_{2}^{j}(\phi)\right) \text { a.e. } t
\end{gathered}
$$

We notice $\left(\alpha\left(\psi^{j}\right)+\left(\psi^{j}-\phi\right) \alpha^{\prime}\left(\psi^{j}\right)\right)\left|\nabla \psi^{j}\right|^{2}$ is weakly lower semi-continuous since $\psi^{j}$ converges strongly in $L^{2}$ a.e. $t$ and $\alpha\left(\psi^{j}\right)+\left(\psi^{j}-\phi\right) \alpha^{\prime}\left(\psi^{j}\right) \geqslant 0$ by the assumption. Therefore,

$$
\begin{aligned}
& \underset{j}{\liminf } \int_{\Omega} \sqrt{\alpha\left(\psi^{j}\right)} \nabla \psi^{j} \cdot \nabla\left(\sqrt{\alpha\left(\psi^{j}\right)}\left(\psi^{j}-\phi\right)\right) \\
& \quad=\liminf _{j} \int_{\Omega}\left(\alpha\left(\psi^{j}\right)+\alpha^{\prime}\left(\psi^{j}\right)\left(\psi^{j}-\phi\right)\right)\left|\nabla \psi^{j}\right|^{2}-\int_{\Omega} \alpha \nabla \psi \nabla \phi \\
& \quad \geqslant \int_{\Omega}\left(\alpha+\alpha^{\prime}(\psi-\phi)\right)|\nabla \psi|^{2}-\int_{\Omega} \alpha \nabla \psi \nabla \phi \quad \text { a.e. } t
\end{aligned}
$$

This proves (34) and completes the proof.

Finally, we also obtain the existence of a measure-valued solution in the following theorem, for $p \geqslant 2(d=2,3)$. 


\section{Theorem 6}

Under the assumptions of Theorem 5, there exists a measured-valued solution $(u, \lambda, \psi, \mu)$ satisfying Definition 2 with (13) replaced by (34).

\section{Proof}

The proof is similar to the one of Theorem 5, replacing $\partial_{t} \boldsymbol{u}^{j} \in L^{1}\left(0, T ;\left(\mathbf{J}^{1, p}\right)^{\prime}\right)$ by $\partial_{t} u^{j} \in$ $L^{p^{\prime}}\left(0, T ;\left(\mathbf{J}^{k, 2}\right)^{\prime}\right), k>1+d / 2$, and arguing as in Theorem 2 .

\section{ACKNOWLEDGEMENTS}

This work was done while the first author visited CMAF, University of Lisbon, with a post-doctoral fellowship. The last author acknowledges partial support of Project POCTI/MAT/34471/2000 of the FCT (Fundação para a Ciência e Tecnologia).

\section{REFERENCES}

1. Helfand E, Fredrickson GH. Large fluctuations in polymer solutions under shear. Physical Review Letters 1989; 62:2468-2471.

2. Koga T, Kawasaki K. Spinoidal decomposition in binary fluids: effects of hydrodynamic interactions. Physical Review A 1991; 44:R817-R820.

3. Onuki A, Kawasaki K. Nonequilibrium steady state of critical fluids under shear flow: a renormalization group approach. Annals of Physics 1979; 121:456-528.

4. Hohenberg PC, Halperin BI. Theory of dynamic critical phenomena. Reviews of Modern Physics 1977; 49: 435-479.

5. Gurtin ME, Polignone D, Viñals J. Two-phase binary fluids and immiscible fluids described by an order parameter. Mathematical Models and Methods in Applied Sciences 1996; 6:815-831.

6. Lowengrub J, Truskinovsky L. Quasi-incompressible Cahn-Hilliard fluids and topological transitions. Royal Society of London Proceedings A 1998; 454:2617-2654.

7. Boyer F. A theoretical and numerical model for the study of incompressible mixture flows. Computer and Fluids 2002; 31:141-168.

8. Anderson D, McFadden GB, Wheeler AA. Diffuse interface methods in fluid mechanics. Annals of Review Fluid Mechanics 1998; 30:139-165.

9. Novick-Cohen A. Energy methods for the Cahn-Hilliard equation. Quarterly Applied Mathematics 1988; 46:681-690.

10. Jacqmin D. Calculation of two-phase Navier-Stokes flows using phase-field modeling. Journal of Computational Physics 1999; 155:96-127.

11. Kim J, Kang K, Lowengrub J. Conservative multigrid methods for Cahn-Hilliard fluids. Journal of Computational Physics 2004; 193:511-543.

12. Boyer F. Non-homogeneous Cahn-Hilliard fluids. Annales de l Institut Henri Poincaré, Anal. non linéaire 2001; 18(2):225-259.

13. Boyer F. Mathematical study of multiphase flow under shear through order parameter formulation. Asymptotic Analysis 1999; 20:175-212.

14. Lions JL. Quelques méthodes de résolution des problèmes aux limites non linéaires. Dunod et Gauthier-Villars: Paris, 1969.

15. Málek J, Nečas J, Rokyta M, Rǔžička M. Weak and Measure-Valued Solutions to Evolutionary PDEs. Chapman and Hall: London, 1996.

16. Blowey JF, Elliot CM. The Cahn-Hilliard gradient theory for phase separation with non-smooth free energy. Part I: mathematical analysis. European Journal of Applied Mathematics 1991; 2:233-280.

17. Kenmochi N, Niezgódka M, Pawlow I. Subdifferential operator approach to the Cahn-Hilliard equation with constraint. Journal of Differential Equation 1995; 117:320-354.

18. Nirenberg L. On elliptic partial differential equations. Annali della Scuola Norm. Sup. Pisa 1959; 13:115-162. 